\section{AB0430 DEVELOP A REPLICABLE MODEL FOR RATIONAL SELECTION OF STRATEGIES IN TREAT-TO-TARGET AND MAINTAIN-BEING-TARGET: REAL WORLD DATA MINING VIA SMART SYSTEM OF DISEASE MANAGEMENT (SSDM)}

Rong Mu ${ }^{1}$, LI Chun ${ }^{1}$, Jing Yang ${ }^{2}$, Xiaohan Wang ${ }^{3}$, Bin Wu ${ }^{4}$, Fengxiao Zhang ${ }^{5}$, Yong Wang $^{6}$, LI Qin $^{7}$, MI Cundong ${ }^{8}$, Huifang Guo ${ }^{9}$, Wei Wei ${ }^{10}$, Wei Liu' ${ }^{11}$, Qingchun Huang ${ }^{12}$, Jing Lu ${ }^{13}$, Yang Liu ${ }^{14}$, LI Huiling ${ }^{15}$, Hui Xiao ${ }^{16}$, Yuhua Jia ${ }^{16}$, Fei Xiao ${ }^{16}$, LI Zhanguo ${ }^{1}$, SSDM Collaboration Group, China. ${ }^{1}$ People's Hospital, Beijing University Medical School, Beijing, China: ${ }^{2}$ Central Hospital of Mian Yang, MianYang, China; ${ }^{3}$ Anyang district hospital, Anyang, China; ${ }^{4}$ Chongqing Hospital of Traditional Chinese Medicine, Chongqing, China; ${ }^{5}$ Hebei General Hospital, Shijiazhuang, China; ${ }^{6}$ The first Hospital Affiliated to AMU (Southernwest Hospital), Chongqing, China; ${ }^{7}$ The No.3 People's Hospital of HuZhou City, Huzhou, China; ${ }^{8}$ The Second Affiliated Hospital of Guangxi Medical University, Nanning, China; ${ }^{9}$ The Second Hospital of Hebei Medical University, Shijiazhuang, China; ${ }^{10}$ Tianjin Medical University General Hospital, Tianjin, China; ${ }^{11}$ The First Teaching Hospital of Tianjin University of Traditional Chinese Medicine, Tianjin, China; ${ }^{12}$ Guangdong Provincial Hospital of Chinese Medicine, Guangzhou, China; ${ }^{13}$ First Affiliated Hospital of China Medical University, Shenyang, China; ${ }^{14}$ Affiliated Hospital of Inner Mongolia Medical University, Hohhot, China; ${ }^{15}$ Hubei Hospital of Traditional Chinese Medicine, Wuhan, China; ${ }^{16}$ Shanghai Gothic Internet Technology Co., Ltd., Shanghai, China

Background: Daily health care in the real world is different from a clinical trial setting, which deals with a wide spectrum of RA patients from statuses of remission to disease activities at mild, moderate and severe based on DAS28. Due to lack of information and knowledge about optimal regiments for both treat-to-target (T2T) and maintain-being-target $(\mathrm{MbT})$, physicians make choices on treatment strategies based on their own experience or intuition.

Objectives: To develop a replicable model for rationalizing the strategies for T2T and MbT using data mining via smart system of disease management (SSDM)

Methods: SSDM is an interactive mobile disease management tool, including two application systems (APPs) for both the doctors and the patients. The patients can input medical records (including medication and laboratory test results) and perform self-evaluation (DAS28, HAQ) via App. The data synchronizes to mobiles of authorized rheumatologists through cloud and advices could be delivered. In previous studies, we demonstrated that patients could master SSDM after training.

Up to January of 2019 , totally 106,647 patients with rheumatic diseases using SSDM, among them, $38 \%$ are RA patients who receive more than 1,400 different regiments of treatments. Here we select MTX, Hydroxychloroquine (HCQ) and prednisone (GS) based therapies for model development.

Results: Totally 1571 patients were treated with MTX (640), HCQ (397), GS (131), MTX+HCQ (253), MTX+GS (47), HCQ+GS (61), MTX+HCQ +GS (42), respectively. Among the patients whose DAS28<3.2 at baseline, $72 \%$ with MTX, $75 \%$ with $\mathrm{HCQ}, 78 \%$ with $\mathrm{MTX}+\mathrm{HCQ}$ and $73 \%$ with $\mathrm{MTX}+\mathrm{HCQ}+\mathrm{GS}$ are MbT (DAS28<3.2) after 6 months, which are significantly different comparing with those $52 \%$ with GS, $57 \%$ with MTX+GS and $57 \%$ with $\mathrm{HCQ}+\mathrm{GS}(\mathrm{p}<0.01)$. Among the patients whose DAS28 $>5.1$ at baseline, $81 \%$ with GS, $72 \%$ with MTX+GS and $100 \%$ with HCQ+GS achieved T2T (DAS28<3.2) after 6 months, which is significantly different compared to $25 \%$ with MTX, $24 \%$ with $\mathrm{HCQ}, 45 \%$ with MTX+HCQ and $37 \%$ with $\mathrm{MTX}+\mathrm{HCQ}+\mathrm{GS} \quad(\mathrm{p}<0.01)$. Among the patients whose $3.2<$ DAS28<5.1 at baseline, there is no statistic significant differences for the rates of achieving T2T, which rates ranging from $39 \%$ to $50 \%$ across all the 7 regiments.

Conclusion: With MTX, HCQ and GS based regiments in RA, the rational selections of strategies for MbT are mono therapy with MTX, $H A Q$, or $M T X+H C Q$, strategies for T2T on high disease activity group are GS, GS +MTX or GS+HCQ. In view of 1400 regiments with clinical outcomes being available in SSDM, the model developments can be replicated in rationalizing strategies through data mining.

Disclosure of Interests: None declared

DOI: 10.1136/annrheumdis-2019-eular.6512

\section{$\mathrm{AB} 0431$ \\ EFFECTS OF LOW-LOAD RESISTANCE EXERCISE OF LOW EXTREMITY IN WOMEN WITH RHEUMATOID ARTHRITIS}

Satoshi Nakazaki ${ }^{1}$, Takashi Murayama ${ }^{2}$, Shinichi Kato ${ }^{3}$, SSDM Collaboration Group, China. ' Johoku Hospital, Rheumatology, Kanazawa, Japan; ${ }^{2}$ Johoku Hospital, Rheumatology, Kanazawa, Japan; ${ }^{3}$ Kamiaraya Clinic, Rheumatology, Kanazawa, Japan

Background: Some skeletal muscles communicate with other organ by secreting proteins and peptides called myokine. Some myokines have anti-inflammatory effect ${ }^{1}$. As regards rheumatoid arthritis (RA), many studies have shown that aerobic and resistance exercise programs do not change the number of inflammatory joints and RA disease activity, whereas other studies showed improved the marker. And almost studies were performed by high-load resistance training protocols. Meanwhile lately Schoenfeild showed that muscle hypertrophy can be equally achieved across a spectrum of load ranges with meta-analysis ${ }^{21}$.

Objectives: To investigate the efficacy of low-load resistance exercise of low extremity protocol on RA.

Methods: Twenty-four patients with RA were enrolled. Inclusion criteria were receipt of a stable dose of biologics, JAK inhibitory, and conventional DMARDs more than 3months prior to the first exercise, and corticosteroid and NSAIDs more than one month prior to the first exercise. The exercise circuits consisted of 6 different low extremity exercises intended to improve arthritis. Within the exercise circuits, each exercise was repeated 8-12 times and less than $50 \%$ of the 1 -repitition maximum The exercise circuits were performed 3 times a week. Disease activity parameters and inflamed joint of upper extremity and lower extremity were collected at base line and 2 months after exercise started. The Wilcoxon signed-rank test was used to examine the difference between the parameter of the base line and the 2 months.

Results: All patients were female. Mean age was 68.7 years., and mean disease duration 15.3 years. Seven patients $(29 \%)$ used methotrexate, 5 patients $(21 \%)$ used prednisolone and 13 patients $(54 \%)$ used biologics. Mean DAS28(ESR) was 3.98 at base line and 3.58 at 2 months, mean DAS28(CRP) 3.46 and 3.06, mean SDAI 15.4 and 12.5, and mean CDA 14.5 and 11.5 , and mean upper extremity joint tenderness 4.7 and 3.7 , mean upper extremity joint swelling 1.6 and 1.8, mean lower extremity joint tenderness 5.8 and 2.2, mean lower extremity joint swelling 4.0 and 2.0 respectively. Every index except upper extremity joint swelling improved significantly $(p<0.05)$ at 2 months than baseline.

Conclusion: Low intensity exercise of low extremity was efficacious against not only lower extremity arthritis but also upper extremity joint arthritis in patients with rheumatoid arthritis

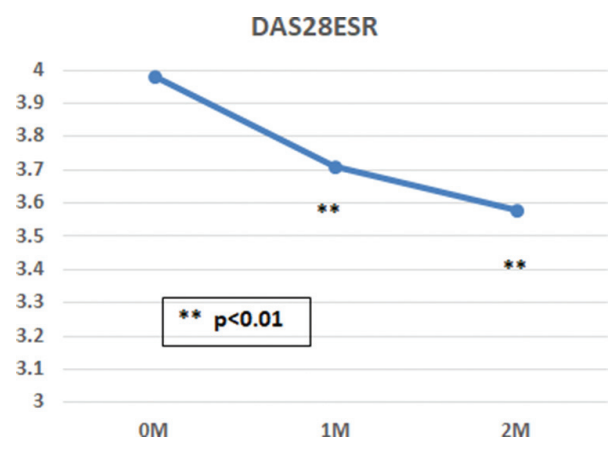

upper extremity joint tenderness

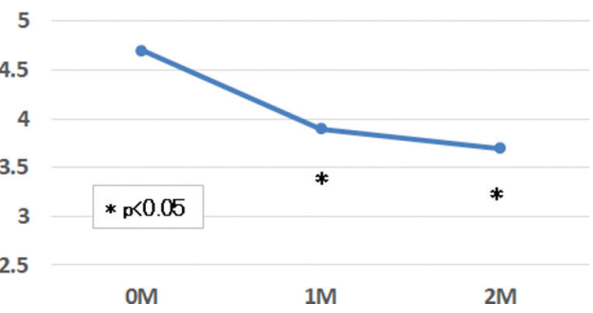

REFERENCES

[1] Fabiana B Benatti, Bente K Pedersen: Exercise as an anti-inflammatory therapy for rheumatic diseases-myokine regulation. Nat Rev Rheumato 11: 86-97, 2015 